\title{
Morphological and Molecular Characterization of Pleurotus pulmonarius Hybrids with Improved Sporophore Features and Higher Biological Efficacy
}

\author{
Rosnina Abdulgani ${ }^{1,2}$, Ching Ching Lau ${ }^{1}$, Noorlidah Abdullah ${ }^{1^{*}}$ and S. Vikineswary ${ }^{1}$ \\ ${ }^{1}$ Mushroom Research Centre, Institute of Biological Sciences, Faculty of Science, University of Malaya, 50603 Kuala Lumpur. \\ Malaysia \\ ${ }^{2}$ Department of Agroecotechnology of Agriculture Faculty, University of Malikussaleh Lhokseumawe, Aceh, Indonesia \\ *For correspondence: noorlidah@um.edu.my
}

\begin{abstract}
To improve the sporophore characteristics and production yield of Pleurotus pulmonarius by interspecies hybridization between monokaryotic cultures of $P$. pulmonarius and $P$. citrinopileatus, a total of a hundred possible pairings were done. Five hybrids were obtained producing sporophores with morphological characteristics dominant to those of $P$. pulmonarius. DNA analysis confirmed the high genetic affiliation of the hybrids with $P$. pulmonarius generating a bootstrap value of $99 \%$. Among the hybrids, P19xC5 strain exhibited good traits such as higher mycelial growth rate, thicker mycelium density as well as generating sporophores with fleshier texture and bigger pileus. Further analysis by spawning showed that it had faster growth rate at $8.7 \mathrm{~mm} /$ day as opposed to 8.2 and $7.7 \mathrm{~mm} /$ day for $P$. pulmonarius and $P$. citrinopileatus, respectively. It generated a higher sporophores yield (182.97 g) and biological efficiency $(130.19 \%)$ which was approximately twice that of the parental strains. Its sporophore was found to contain lower spore density, a criteria favourable for the health and work conditions of farm workers. The good features imposed by the hybrid P19xC5 may indicate its better adoption in the mushroom growing industry and greater commercialization value. (C) 2017 Friends Science Publishers
\end{abstract}

Keywords: Pleurotaceae; Mushroom breeding; Sporophore yield; Biological efficiency

\section{Introduction}

To date, interspecies mating of cultivated edible fungi has been carried out on laboratory scale to breed new varieties of mushrooms with better traits. Successful approaches had been documented in several studies with a majority of the research revolved around the genus Pleurotus (Gupta et al., 2011; Adebayo et al., 2013; Liu et al., 2016). Apart from their high nutritional and medicinal values, the popularity of Pleurotus spp. in the mushroom industry attributed primarily for the ability to grow on a large range of substrate and climate condition (Chang, 2008). The two Pleurotus spp. tested in this work have different features that are unique to the species.

P. pulmonarius (Fr.) Quél (1827) or commonly known as grey oyster mushroom has a grayish coloured sporophore with a fleshy texture and produce an aromatic, not anise-like aroma (Lechner and Wright, 2004). The pileus (5-20 cm in diameter) is convex, lung-shaped and often wavy with age. The stems are eccentrically attached to the cap (Lechner and Wright, 2004). It rarely grows in clusters of more than 5 mushrooms (Stamets, 1993). Contrary, P. citrinopileatus, or yellow oyster mushroom has a golden to bright yellow coloured cap with an extremely fragile flesh. It imparts a unique nutty scent that is distinctly recognizable to this species. The pileus $(2-5 \mathrm{~cm}$ in diameter) is convex with a centrally attached stipe. It grows in large clusters, arising from a single, joined base (Stamets, 1993).

The present study aimed to produce a new Pleurotus variety with fleshy pileus and rigid stipe as those of $P$. pulmonarius along with cluster-type growing pattern akin to $P$. citrinopileatus. This type of growth trend promises higher total sporophore yield hence, better biological efficiency. In this study, the morphological and molecular characteristics of the hybrids produced by the interspecies crosses of hyphal fusion between $P$. pulmonarius and $P$. citrinopileatus were investigated and compared with those of the parental strains. Finally, a strain with the best feature was analyzed for its potential as a new variety of mushroom for commercial production.

\section{Materials and Methods}

\section{Isolation of Single Basidiospores}

Single basidiospore isolation was done according to Gupta et al. (2011) with slight modifications. First, a small piece of pileus $(1 \mathrm{~cm} \times 1 \mathrm{~cm})$ was attached with Vaseline to the

To cite this paper: Abdulgani, R., C.C. Lau, N. Abdullah and S. Vikineswary, 2017. Morphological and molecular characterization of Pleurotus pulmonarius hybrids with improved sporophore features and higher biological efficacy. Int. J. Agric. Biol., 19: 707-712 
inside of a Petri dish lid hanging the gill side down above a solidified malt extract agar (MEA, Oxoid, Cat. No. CM0059). The plate was incubated at $25^{\circ} \mathrm{C}$ in a sloping position for three to four hours upon which the pileus was removed. Further incubation for two to three days generates spore germination. A single spore was picked off manually using a fine needle with the aid of a microscope under 40X magnifications and it was left to grow on a fresh MEA plate for 7 days at $25^{\circ} \mathrm{C}$. The mycelium was confirmed as monokaryon by the absence of clamp connections.

\section{Hybridization of Monokaryon Cultures}

Ten monokaryon cultures from parental $P$. pulmonarius (designated as P1 through $\mathrm{P} 10$ ) and $P$. citrinopileatus (designated as $\mathrm{C} 1$ through $\mathrm{C} 10$ ) were paired against each other in every possible combination between the species generating a total of one hundred possible crosses using two point inoculation technique by Gupta et al. (2011). In this method, plug mycelium $(0.7 \mathrm{~cm}$ diameter $)$ from monokaryon cultures of two different parental mushrooms were transferred about $3 \mathrm{~cm}$ apart from each other in a single fresh MEA plate and incubated at $25^{\circ} \mathrm{C}$ for one week. Each pairing was carried out in triplicates and compatible mating was recognized by little or no line formation at the confrontation zone of the two mycelia cultures. Mycelial culture of compatible mated pair (i.e. strains from $\mathrm{P} 9 \mathrm{xC7}$, $\mathrm{P} 13 \mathrm{xC} 5, \mathrm{P} 13 \mathrm{xC} 7, \mathrm{P} 19 \mathrm{xC} 2$ and $\mathrm{P} 19 \mathrm{xC} 5)$ was subjected to further analysis.

\section{Evaluation of Growth Rate and Thickness of the Selected Mycelial Culture Inoculated on the MEA Plate}

Plug mycelium $(0.7 \mathrm{~cm}$ diameter $)$ from the interface zone of each compatible mated culture was transferred onto a fresh MEA plate and left to grow at $25^{\circ} \mathrm{C}$. Dikaryons from $P$. pulmonarius and $P$. citrinopileatus were grown alongside the hybrid strains. The diameter of the resulting mycelia colony was measured in two perpendicular directions every day until they attained the full growth in the $90 \mathrm{~cm}$ Petri dish. The average reading was plotted against time (day) to obtain the growth rate in $\mathrm{mm} /$ day. Mycelia thickness was also observed and categorized based on the indicator by Razak et al. (2013). All the strains were tested in triplicates.

\section{Production of Sporophores from the Parental and Hybrid Strains of $P$. pulmonarius and $P$. citrinopileatus for Morphological Characterization}

The fruiting substrate consists of sawdust supplemented with $10 \%$ rice bran $(\mathrm{w} / \mathrm{w}), 2 \%$ calcium carbonate $(\mathrm{w} / \mathrm{w})$ and $80 \%$ of moisture content (v/w). The sterilized substrate bags $(600 \mathrm{~g})$ were inoculated with three to five plugs of seven days old mycelium discs $(0.7 \mathrm{~cm}$ diameter) from the hybrids or parental strains. Three bags were prepared for each strain. The inoculated bags were kept at $27 \pm 1^{\circ} \mathrm{C}$ to allow spawn run. Thereafter, all the bags were opened to induce fruiting and placed in the mushroom house with ample ventilation and humidity at $25^{\circ} \mathrm{C}$. The environment was watered two to three times every day during the fruiting phase. Matured sporophores generated were evaluated for their morphological characteristics, i.e. colour, texture and aroma.

\section{Phylogenetic Analysis of the Mushroom Sporophores}

Genomic DNA was extracted from dried herbarium specimen using an E.Z.N.A ${ }^{\circledR}$ Forensic DNA Kit (Omega 118 Bio-tek Inc. Norcross, GA, USA) following the manufacturer's protocol. The internal transcribed spacers (ITS-1 and ITS-2, including the 5.8S rRNA) were amplified using primer pairs ITS-1 and ITS-4. Polymerase Chain Reaction (PCR) was carried out using $i$-Taq ${ }^{\mathrm{TM}}$ plus DNA Polymerase kit (iNtRON Biotechnology, Inc., Gyeonggi-do, Korea). Each reaction tube contained $1 \mathrm{X} i$-Taq ${ }^{\mathrm{TM}}$ plus PCR buffer, 1 unit $i$-Taq ${ }^{\mathrm{TM}}$ plus DNA polymerase, $0.2 \mathrm{mM}$ of each dNTP, $0.5 \mu \mathrm{M}$ ITS-1, $0.5 \mu \mathrm{M}$ ITS-4 and $1 \mu \mathrm{L}$ DNA template. The mixture was topped up to $50 \mu 1$ with sterile distilled water. The reactions were performed on a Thermal Cycler (MyCycler ${ }^{\mathrm{TM}}$ Thermal Cycler, BioRad Laboratories, Inc., Hercules, USA) with the following profile: initial denaturation at $94^{\circ} \mathrm{C}$ for $5 \mathrm{~min}, 30$ cycles of denaturation at $94^{\circ} \mathrm{C}$ for $1 \mathrm{~min}$, annealing at $48^{\circ} \mathrm{C}$ for $1 \mathrm{~min}$, extension at $72^{\circ} \mathrm{C}$ for $1 \mathrm{~min}$, final extension at $72^{\circ} \mathrm{C}$ for $10 \mathrm{~min}$ and a hold at $4^{\circ} \mathrm{C}$. Amplified DNA fragments were resolved on a $1 \%$ agarose gel by electrophoresis separation at $80 \mathrm{~V}$. The resulting DNA fragment was purified with MEGAquick$\operatorname{spin}^{\mathrm{TM}}$ Total Fragment DNA Purification Kit (iNtRON Biotechnology, Inc., Gyeonggi-do, Korea), following the manufacturer's protocol. Purified PCR products were then sent to Bioneer Corporation, Korea for sequencing. Raw sequences obtained were checked manually and edited using the Chromas Lite, version 2.1.1. Consensus sequences were aligned with the Clustal $\mathrm{W}$ algorithm of the MEGA5 software (Tamura et al., 2011). Phylogenetic tree was constructed using the neighbour-joining method, with the distances estimated by the Kimura 2-parameter model and a bootstrapping of 1000 replications.

\section{Evaluation of the Radial Growth Rate in Bag, Sporophore Yield and Biological Efficacy of the Parental Mushrooms and Selected Hybrid P19xC5}

Hybrid strain of P19xC5, which has good characteristics such as higher mycelia growth rate and thick mycelium density, as well as sporophores with fleshier texture, bigger pileus and nice aroma was selected for the current analysis. Cultivation was carried out as described in the section above and 100 bags were prepared for each parental strain and hybrid $\mathrm{P} 19 \mathrm{xC} 5$. The radial growth rate during spawn running was determined by measuring the mycelia extension at four sides of the bag every two days until the spawn run completed. The average reading was plotted 
against time (day) to obtain the growth rate in $\mathrm{mm} /$ day. Matured sporophores were picked up every two to four days during the fruiting phase. The first three flushes harvested were accounted for the total sporophores yield for each strain. At the end of third flush, biological efficiency of fruiting substrate was calculated following the formula as below:

$$
\text { Biological efficiency }(\%)=\frac{\text { Total weight of fresh sporophores }(\mathrm{g})}{\text { Dry weight of substrate }(\mathrm{g})} \times 100
$$

Comparison of Spore Density between Parental $P$. pulmonarius and the Selected Hybrid P19xC5

Matured sporophore was used for the spore density analysis using a spore print method. First, the stipe was removed from the sporophore of $P$. pulmonarius and hybrid P19xC5. The gills were placed downward on a piece of art paper and left overnight.

\section{Phenotypic Evaluation of the Hybrid P19xC5 Sporophore Grown for Three Generations}

Mycelial colony was generated from the matured sporophore of P19xC5 (first generation) by tissue culture method. Three to five mycelium plugs $(0.7 \mathrm{~cm})$ from the dikaryon culture were inoculated in the fruiting substrate and cultivation was carried out as described in the section above. Mycelial culture was acquired from the matured sporophore (second generation) and inoculated again into fresh substrate bags. The sporophore attained was designated as third generation. Three bags were prepared for each generation. Matured sporophore produced was compared for phenotypic evaluation.

\section{Statistical Analysis}

Quantitative data such as radial growth rate of mycelia, production yield of sporophores, and biological efficiency were carried out at least in triplicates. Mean differences were analyzed using one-way ANOVA in Statgraphics Plus 3.0 at $\mathrm{p}<0.05$.

\section{Results}

Cultural Characteristics and Sporophore Morphology of Parental and Hybrid Strains of $P$. pulmonarius and $P$. citrinopileatus

From the results shown in Table 1, there was a significant difference in the mycelial growth rate between the hybrids and their parental strains. Both the parent $P$. pulmonarius and $P$. citrinopileatus recorded a similar growth rate at 2.3 $\mathrm{mm} /$ day. In contrast, the hybrid strains showed an exceptionally faster degree of expansion with their velocity descend in the following order: P19xC5 (4.0 mm/day) > $\mathrm{P} 13 \mathrm{xC} 7$ (3.6 mm/day) > P13xC5 (3.3 mm/day) > P9xC7
(3.2 $\mathrm{mm} /$ day) > P19xC2 (3.0 mm/day). The mycelial mat for the hybrid strains were generally denser than the parents with the thickest being observed in $\mathrm{P} 9 \mathrm{xC} 7, \mathrm{P} 13 \mathrm{xC} 5$, $\mathrm{P} 13 \mathrm{xC} 7$ and $\mathrm{P} 19 \mathrm{xC} 5$. In general, sporophores of the hybrid strains showed morphological characteristics dominant to $P$. pulmonarius (Fig. 1, Table 1). Hybrids P13xC5 and P19xC5 had a similar grey colour as $P$. pulmonarius, however, their pileus were thicker and fleshier. Among the two hybrids, the former had a relatively smaller mushroom cap diameter and shorter stipe length as compared to the latter. Literally, the pileus width of $\mathrm{P} 19 \mathrm{xC} 5$ was even larger than the parent $P$. pulmonarius. Strains $\mathrm{P} 9 \mathrm{xC7}$ and $\mathrm{P} 13 \mathrm{xC} 7$ were shaded in light grey and beige colours, respectively. They had pileus thickness akin to $P$. pulmonarius. Sporophores of $\mathrm{P} 19 \mathrm{xC} 2$ had ivory colour and thin texture. All the hybrids produced a mild and pleasant smell. They were seen growing in clusters larger than those of $P$. pulmonarius.

\section{Phylogenetic Analysis of the Mushroom Sporophore}

Based on the result shown in Fig. 2, the five hybrids stand in the same clade with their parent, $P$. pulmonarius with a bootstrap value at $99 \%$. This may indicate that there is a high genetic homology between the hybrid strains with $P$. pulmonarius. Interestingly, the hybrids were phylogenetically closer to the $P$. eryngii and $P$. ostreatus than their other parental strain, $P$. citrinopileatus.

Evaluation of the Growth Rate in Bag, Sporophore Yield and Biological Efficiency of the Parental Mushrooms and Selected Hybrid P19xC5

In the current study, the hybrid strain P19xC5 exhibited a higher radial growth rate at $8.7 \mathrm{~mm} /$ day, as opposed to 8.2 and $7.7 \mathrm{~mm} /$ day for $P$. pulmonarius and $P$. citrinopileatus, respectively (Table 2). Furthermore, it has increased total sporophores yield (182.97 g) and biological efficiency (130.19\%) which was approximately twice the amount reported for the parental strains. These characteristics are advantageous for the mushroom growing industry.

Comparison of Spore Density between Parental $P$. pulmonarius and the Selected Hybrid P19xC5 and Phenotypic Evaluation of the Hybrid Sporophore Grown for Three Generations

The hybrid strain P19xC5 was further analysed for its spore density in comparison with the commonly cultivated grey oyster mushroom. The result obtained showed the P19xC5 strain generated a significantly lower spore density than $P$. pulmonarius (Fig. 3A). Phenotypic evaluation of the hybrid strain was carried out for three generations to confirm the consistency of the sporophore characteristics. As shown in Fig. 3B, all the three generations of hybrid P19xC5 had consistence appearance, i.e. crowded lamella, big and thick pileus cap and dark grey circle with wavy edge pileus. They produce a mild and pleasant smell with less spore formation. 
Table 1: Comparison of the mycelial growth rate (mm/day) and mat thickness of the parental and hybrid strains of $P$. pulmonarius and $P$. citrinopileatus inoculated on the malt extract agar plate together with their sporophore macromorphology characterization

\begin{tabular}{llllll}
\hline Strain & \multicolumn{2}{c}{ Mycelial } & & Sporophore \\
\cline { 2 - 6 } & ${ }^{*}$ Growth rate $(\mathrm{mm} /$ day) & ${ }^{*}$ Thickness & Colour & Texture & Aroma \\
\hline Parent P. pulmonarius & $2.3 \pm 0.2^{\mathrm{a}}$ & ++ & Grey & Fleshy & Mild \\
Parent P. citrinopileatus & $2.3 \pm 0.3^{\mathrm{a}}$ & + & Yellow & Fragile & Nutty \\
P9xC7 & $3.2 \pm 0.3^{\mathrm{b}}$ & +++ & Light grey & Fleshy & Mild \\
P13xC5 & $3.3 \pm 0.3^{\mathrm{b}}$ &.$++^{\mathrm{b}}$ & Grey & Fleshier & Mild \\
P13xC7 & $3.6 \pm 0.0^{\mathrm{d}}$ & +++ & Beige & Fleshy & Mild \\
P19xC2 & $3.0 \pm 0.2^{\mathrm{d}}$ & ++ & Ivory & Thin & Mild \\
P19xC5 & $4.0 \pm 0.2^{\mathrm{e}}$ & +++ & Grey & Fleshier & Mild \\
\hline
\end{tabular}

"Values are expressed as mean \pm standard deviation $(n=3)$. Mean values with different alphabet letters are significantly different $(p<0.05)$

${ }^{* *}$ The degree of mycelia thickness on agar plate was indicated as follow: ++ ' marked the lowest degree of mycelia thickness; ;++' marked the intermediate degree of mycelia thickness; '+1+' marked the highest degree of mycelia thickness

Table 2: Comparison of the mycelia growth rate in bag, production and biological efficiency of parental strains and selected hybrid (P19xC5)

\begin{tabular}{llll}
\hline Strain & Mycelia growth rate in bag $(\mathrm{mm} /$ day $)$ & ${ }^{*}$ Total sporophores yield $(\mathrm{g})$ & ${ }^{* *}$ Biological efficiency $(\%)$ \\
\hline Parent $P$. pulmonarius & $8.2 \pm 0.1^{\mathrm{a}}$ & $103.9 \pm 0.5^{\mathrm{a}}$ & $74.2 \pm 0.4^{\mathrm{a}}$ \\
Parent $P$. citrinopileatus & $7.7 \pm 0.1^{\mathrm{b}}$ & $71.3 \pm 1.2^{\mathrm{b}}$ & $50.9 \pm 0.3^{\mathrm{b}}$ \\
Hybrid P19xC5 & $8.7 \pm 0.0^{\mathrm{c}}$ & $183.0 \pm 0.2^{\mathrm{c}}$ & $130.2 \pm 0.7^{\mathrm{c}}$ \\
\hline Vax
\end{tabular}

Values are expressed as mean \pm standard deviation $(n=3)$. Mean values with different alphabet letters are significantly different $(\mathrm{p}<0.05)$

"The total sporophores yield was acquired by the total weight of fresh mushroom harvested in the first three flushes

***Bological efficiency was determined by the percentage of total sporophores yield divide by the weight of dry substrate

\section{Discussion}

The hybrid strains from an interspecies mating of $P$. pulmonarius and $P$. citrinopileatus presented a mycelial morphology of cottony texture with variations in density and growth velocity when inoculated in MEA plates (Table 1). Mycelial with a faster rate of radial growth and better colony characteristics are the morphological markers of a good mushroom breed (Gupta et al., 2011). The former criteria are essential to ensure faster substrate colonization, resulting in more rapid completion of the production cycle, thus expedite the time to fructify (Yang et al., 2013). On the other hand, the thicker mycelium mat provides better ability to colonize vast agricultural lignocellulosic wastes. Utilization of these by-products for the production of oyster mushrooms is deemed more feasible and economical (Mandeel et al., 2005).Visual assessment of morphological characteristic of the mushroom sporophores showed the hybrid strains present phenotypes dominant to $P$. pulmonarius (Fig. 1; Table 1). Further analysis via DNA molecular work confirmed the high genetic homology between the five hybrids with P. pulmonarius (Fig. 2). Moreover, akin to $P$. pulmonarius, the hybrids exhibited a closer phylogenetic affiliation to $P$. ostreatus and $P$. eryngii than to their other parental strain, $P$. citrinopileatus. The proximate genetic relationship among the $P$. pulmonarius, $P$. ostreatus and $P$. eryngii is well documented (Bao et al., 2004). Based on these observations, it is possible to suggest that the five hybrids have a highly distinctive morphological features and DNA sequences from $P$. citrinopileatus.

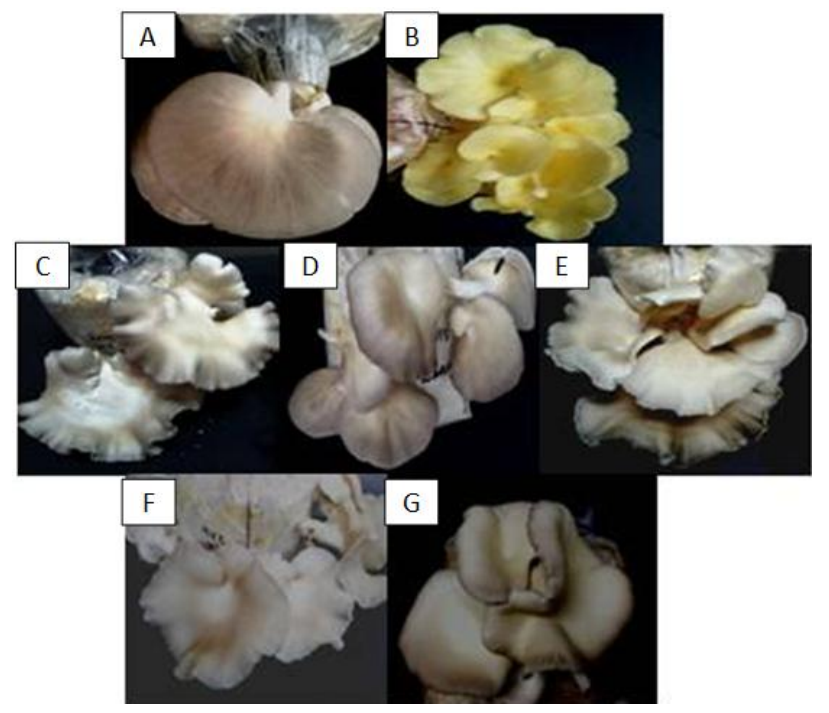

Fig. 1: Sporophores of (A) parent P. pulmonarius, (B) parent $P$. citrinopileatus, (C) Hybrid P9xC7 (D) Hybrid P13xC5 (E) Hybrid P13xC7 (F) Hybrid P19xC2 and (G) Hybrid P19xC5

Among the five hybrids, P19xC5 had the characteristics required in a good mushroom breed, i.e. high mycelia growth rate, thick colony, and produced sporophores with fleshier texture, bigger pileus and nice aroma. Consequently, it was selected for further evaluation of its potential as a new variety of mushroom for commercial production. The $\mathrm{P} 19 \mathrm{xC} 5$ retain its high radial growth rate when inoculated in substrate bags (Table 2). 


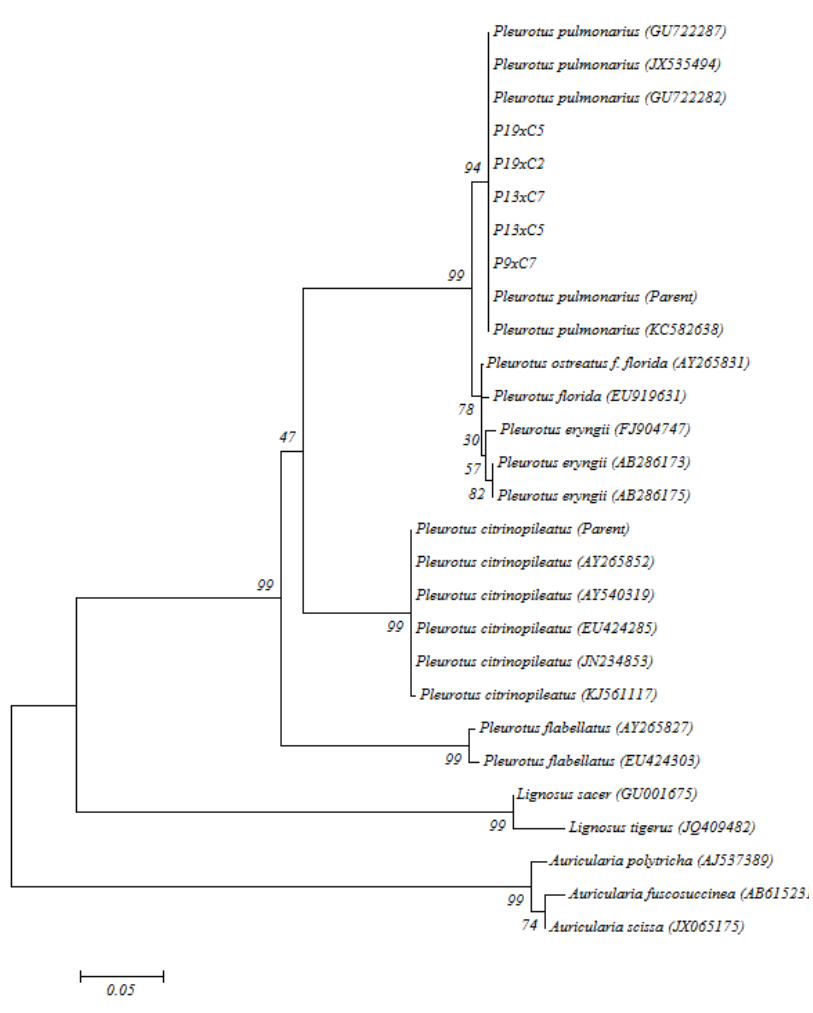

Fig. 2: Phylogenetic tree analysis of DNA gene sequences constructed by neighbour-joining tree using Tamura 3parameter model

Bootstrap values are expressed as percentages support from 1000 replicates showing the similarity among the five hybrids (P9xC7, P13xC5, P13xC7, $\mathrm{P} 19 \mathrm{xC} 2$ and $\mathrm{P} 19 \mathrm{xC5}$ ) with parental strains and the homogenous strains of Pleurotus species

It took a shorter time to fully colonize the spawn, thereby fructify faster. Moreover, the hybrid strain had a high total sporophore production and an excellent biological efficiency, thus ensure its wide acceptance by the mushroom growers as these criteria promise higher income and comparatively more cost-effective. It was noted that environmental conditions and substrate composition could impact the growth, yield and quality of mushrooms (Yang et al., 2013). Hence, this may suggest possible further improvement of the P19xC5 biological efficiency via the optimization of the culture conditions.

The high spore production of mushrooms is a recognized issue. Accumulation of heavy spore load on farms has been associated with the decline in yield and quality of successive crops (Baars and Hesen, 2008). Besides, Cox and co-researchers have proclaimed the mushroom spores as the causal agent responsible for the development of chronic cough and respiratory allergic symptoms in many mushroom growers (Cox et al., 1988). In severe cases, some were found contracting lung damage, rendering the person permanently disabled (Baars and Hesen, 2008). In our study, spore print analysis showed the mushroom cap of P19xC5 generated
A

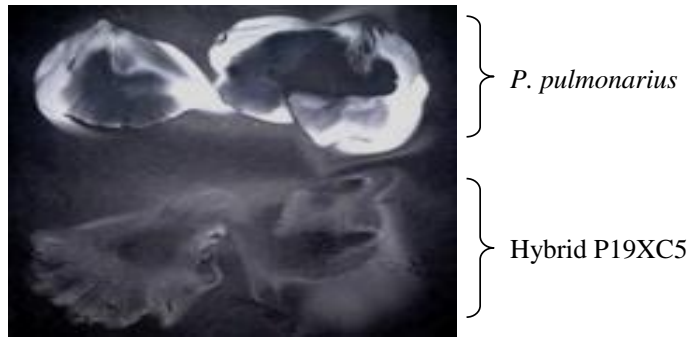

B

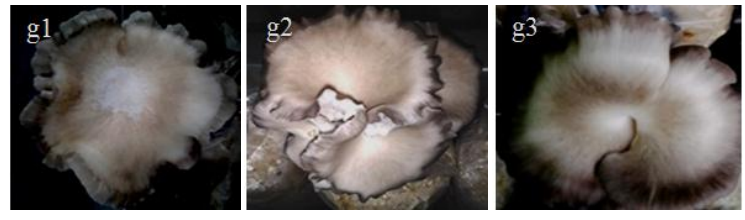

Fig. 3: (A) Comparison of the spore print on art paper for parent $P$. pulmonarius (upper panel) and hybrid strain P19xC5 (lower panel) and (B) the sporophores for the hybrid strain $\mathrm{P} 19 \mathrm{xC} 5$ produced in three generations (g1, g2 and g3)

lesser spores as compared to those from the $P$. pulmonarius (Fig. 3A). Obviously, the cultivation of $\mathrm{P} 19 \mathrm{xC5}$ is beneficial for the health and work conditions of farm workers.

\section{Conclusion}

Present findings revealed that the hybrid P19xC5 could serve as a new mushroom variety for commercial cultivation. It contains superior sporophore features of $P$. pulmonarius (fleshy, big pileus, rigid stipe) and $P$. citrinopileatus (cluster-type growing pattern). Besides, it had shorter spawn run period, produced a higher sporophore yield and increased biological efficiency as compared to the two parental strains. Its lower spore formation should attract the interest of mushroom growers to use it as a substitute for P. pulmonarius.

\section{Acknowledgments}

The authors would like to thank Glami Lemi Biotechnology Research Centre (PPBGL), University of Malaya for the facilities and for the grants IPPP/UPDiT/Geran (PPP) S239/2008 C and Frontier Science UMRG: RP015A-14AFR.

\section{References}

Adebayo, E.A., J.K. Oloke, A. Yadav, M. Barooah and T.C. Bora, 2013. Improving yield performance of Pleurotus pulmonarius through hyphal anastomosis fusion of dikaryons. World J. Microbiol. Biotechnol., 29: 1029-1037

Baars, J., and H. Hesen, 2008. Experience with sporeless strains of oyster mushroom (Pleurotus ostreatus) in commercial production. Proceedings of the $17^{\text {th }}$ Congress of the International Society for Mushroom Science, pp: 774-913 
Bao, D., H. Ishihara, N. Mori and Y. Kitamoto, 2004. Phylogenetic analysis of oyster mushrooms (Pleurotus spp.) based on restriction fragment length polymorphisms of the 5' portion of 26S rDNA. J. Wood Sci., 50: $169-176$

Chang, S.T., 2008. Overview of mushroom cultivation and utilization as functional foods. In: Mushrooms as Functional Foods, pp: 1-33. Cheung, P.C.K. (ed.). John Wiley \& Sons Inc., New York, USA

Cox, A., H.T.M. Folgering and V.G. L.J.L.D., 1988. Extrinsic allergic alveolitis caused by spores of the oyster mushroom Pleurotus ostreatus. Eur. Respir. J., 1: 466-468

Gupta, B., B.P.N. Reddy and A.S. Kotasthane, 2011. Molecular characterization and mating type analysis of oyster mushroom (Pleurotus spp.) using single basidiospores for strain improvement. World J. Microbiol. Biotechnology, 27: 1-9

Lechner, B.E. and J.E. Wright, 2004. The genus Pleurotus in Argentina. Mycologia, 96: 845-858

Liu, S., X. Wu, X. Liu and B. Ke, 2016. Correlation between mating compatibility and the phylogenetic relationship of a rare edible mushroom, Pleurotus nebrodensis, with different Pleurotus species. Int. J. Agric. Biol., 18: 198-203
Mandeel, Q.A., A.A. Al-Laith and S.A. Mohamed, 2005. Cultivation of oyster mushrooms (Pleurotus spp.) on various lignocellulosic wastes. World J. Microbiol. Biotechnol., 21: 601-607

Razak, D.L.A., N. Abdullah, N.M.K. Johari and V. Sabaratnam, 2013. Comparative study of mycelia growth and sporophore yield of Auricularia polytricha (Mont.) Sacc on selected palm oil wastes as fruiting substrate. Appl. Microbiol. Biotechnol., 97: 3207-3213

Stamets, P., 1993. Growing Gourmet and Medicinal Mushrooms, $3^{\text {rd }}$ edition. Ten Speed Press, Berekely, California, USA

Tamura, K., D. Peterson, N. Peterson, G. Stecher, M. Nei and S. Kumar, 2011. MEGA5: Molecular evolutionary genetics analysis using maximum likelihood, evolutionary distance and maximum Parsimony methods. Mol. Biol. Evol., 10: 2731-2739

Yang, W., F. Guo and Z. Wan, 2013. Yield and size of oyster mushroom grown on rice/wheat straw basal substrate supplemented with cotton seed hull. Saudi. J. Biol. Sci., 20: 333-338

(Received 15 November 2016; Accepted 15 December 2016) 